\title{
Valor preditivo do teste tuberculínico padronizado em crianças vacinadas com $B C G^{*}$
}

\section{The predictive value of the standard tuberculin test in BCG-vaccinated children}

\section{Gilberto Ribeiro Arantes"}

\begin{abstract}
ARANTES, G.R. Valor preditivo do teste tuberculinico padronizado em crianças vacinadas com BCG. Rev. Saúde públ., S. Paulo, 26: 264-8 , 1992. A aplicabilidade do teste tuberculínico em crianças menores de 5 anos vacinadas com BCG é assunto controvertido. Visando contribuir para esclarecê-lo foi analisado o valor preditivo positivo do teste tuberculínico padronizado em população sob elevada cobertura vacinal e baixa prevalência de infecção tuberculosa. A partir da proporção de reatores fortes em lactentes e escolares vacinados e não vacinados, foram calculadas a razão de declínio da alergia tuberculínica nos vacinados e a razão de crescimento nos não vacinados, o que possibilitou a estimativa dos respectivos valores nas idades intermediárias. A expectativa de falsos-positivos (FP) foi então calculada por diferença. Conhecidas a sensibilidade e a especificidade do teste (E=1-FP), a cobertura BCG e a prevalència de infecção, os valores preditivos (para a infecção tuberculosa) foram: $1,52 \%, 4,22 \%, 8,26 \%$, $14,86 \%$ e $23,00 \%$, do primeiro ao quinto ano de vida. Nessas condiçōes, a probabilidade de uma reação forte ser devida ao BCG é grande, especialmente nos dois primeiros anos, o que reduz a aplicabilidade clínica e epidemiológica do teste.
\end{abstract}

Descritores: Teste tuberculínico, normas. Valor de predição dos testes. Vacina BCG. Tuberculose infantil, epidemiologia.

\section{Introdução}

Estudos epidemiológicos realizados na década de 50 demonstram que a quimioprofilaxia com hidrazida é muito eficaz no sentido de diminuir o risco de complicações extra-pulmonares da primo-infecção tuberculosa, inclusive nos assintomáticos que apresentam determinadas alterações ao exame radiológico do tórax ${ }^{15}$.

A identificação da primo-infecção tuberculosa assintomática, na ausência de contato conhecido com uma fonte bacilifera, era efetuada pelo uso rotineiro do teste tuberculínico nos primeiros anos de vida ${ }^{l}$; e a indicaçāo de quimioprofilaxia ou de tratamento era decidida pelo exame radiológico.

Com a introdução da vacina BCG intradérmica, no calendário oficial de imunizaçōes, diminuiu sensivelmente a necessidade da quimioprofilaxia, uma vez que a vacinação leva aos mesmos resultados, embora agindo por

* Apresentado no $2^{2}$ Congresso Brasileiro de Saúde

Coletiva $/ 3^{2}$ Congresso Paulista de Saúde Pública, São Paulo, 1989.

** Departamento de Epidemiologia da Faculade de Saúde Pública da USP - Av. Dr. Amaldo, 715 - 01246-904 -

São Paulo, SP - Brasil.

Publicação financiada pela FAPESP. Processo Saúde Coletiva 91/4994-0 mecanismo diverso; além do mais, devido à indução de alergia tuberculínica relativamente intensa e duradoura ${ }^{10}$, a vacinação em larga escala comprometeu seriamente a utilização do teste tuberculínico tanto em clínica individual como em saúde pública, a ponto de constar no Manual de Normas Técnicas da Divisão Nacional de Pneumologia Sanitária do Ministério da Saúde ${ }^{14}$, que "nos vacinados com BCG a prova tuberculínica perde seu significado".

Contrastando com esse ponto de vista, entendem outros que, sendo a alergia pós-vacinal menos expressiva nas crianças vacinadas no primeiro mês de vida, em comparação com a hiperergia que caracteriza a tuberculose primária, o teste não teria perdido o significado. $\mathrm{Na}$ opinião de Joncas e col. ${ }^{11}$ "uma reação maior do que 10 a $12 \mathrm{~mm}$ não pode ser atribuída à vacinação com BCG (escarificação) sendo altamente sugestiva de super-infecção com M.tuberculosis". Para Lifschitz ${ }^{12}$, o teste tuberculínico com 5 U.T. de PPD, em crianças vacinadas no período neonatal (por multipuntura) é factível e prático como método para o rastreio da tuberculose, dado que uma reação igual ou superior a $10 \mathrm{~mm}$, depois do primeiro ano de vida, é muito significante e improvavelmente devida ao BCG. 
Acontece que a maior ou menor probabilidade de uma reação forte ser devida à infecção natural e não ao BCG vai depender da força de predição do teste tuberculínico padronizado em relação à infecção tuberculosa, a qual é diretamente proporcional à prevalência da infecção tuberculosa na população e inversamente proporcional à magnitude da presença de falsos-positivos devidos ao BCG. Com o que, seja para fins epidemiológicos, seja para fíns clínicos, a decisão quanto à sua aplicabilidade poderá variar de uma para outra realidade epidemiológica.

Considerando as controvérsias existentes e dada a importância do assunto, o presente trabalho foi idealizado com o objetivo de estimar o valor preditivo ${ }^{21}$ do teste tuberculínico padronizado em relação à infecção tuberculosa e definir sua aplicabilidade em regiōes sob elevada cobertura por BCG intradérmico, nas quais a prevalência da infecção seja muito baixa, como é o caso, por exemplo, da cidade de São Paulo.

\section{Metodologia}

O valor preditivo do teste positivo é calculado com o auxílio de uma tabela $2 \times 2$ :

\begin{tabular}{|c|c|c|c|}
\hline \multirow[b]{2}{*}{$\begin{array}{l}\text { Resultado } \\
\text { do Teste }\end{array}$} & \multicolumn{2}{|c|}{ Diagnóstico } & \multirow[b]{2}{*}{ Total } \\
\hline & Infectado & Não Infectado & \\
\hline Positivo & a & b & $a+b$ \\
\hline Negativo & $c$ & d & $c+d$ \\
\hline Total & $a+c$ & $b+d$ & $n$ \\
\hline
\end{tabular}

Onde: " $\mathrm{a}$ ", infectados detectados pelo teste; "b", falsos-positivos; "c", falsos-negativos; " $\mathrm{d}$ ", não-infectados negativos ao teste; a + c, total de infectados testados; $b+d$, total de não-infectados testados; $a+b$, total de testes positivos; $c+d$, total de testes negativos; " $n$ ", total de indivíduos examinados.

A sensibilidade é dada por $S=(a / a+c) \times 100$ e a especificidade, $E=(d / b+d) X 100$. $O$ valor preditivo do teste positivo $\mathrm{VP}+=(\mathrm{a} / \mathrm{a}+\mathrm{b}) \times 100$ expressa a probabilidade de um indivíduo da população ter a infecção se o seu resultado for positivo: $P\left\{I / T^{+}\right\}$. Finalmente, o valor preditivo do teste negativo, $V P-=(d / c+d) \times 100$ expressa a probabilidade de um indivíduo da população não ter a infecção se o seu resultado for negativo: $P\left\{\overline{\mathrm{I}} / \mathrm{T}^{-}\right\}$.

Quanto maior a sensibilidade menor a ocorrência de falsos-negativos $(S=1-\mathrm{FN})$; quanto maior a especificidade, menos falsospositivos $(E=1-F P)$. Enquanto que "S" e " $E^{\prime \prime}$ dependem intrinsecamente das características técnicas do teste, inclusive o erro experimental, os valores preditivos dependem também da prevalência de infectados na população estudada bem como da prevalência de falsos positivos, seja por infecções atípicas, seja por vacinação com $B C G$.

A população de estudo foi uma população simulada com caracaterísticas semelhantes às da população da Zona Leste da Capital de São Paulo em termos de prevalência de infecção tuberculosa e cobertura BCG, entre 1980 e 1988.

Para preencher a tabela e calcular os valores preditivos positivos (VP+) desejados, foi observada a seguinte seqüência de procedimentos:

\section{Cálculo dos falsos-positivos e sua evoluçāo}

Considerando que os reatores fortes devidos à vacina atuam como falsos-positivos (casela " $b$ ") estes foram estimados, do primeiro até o oitavo ano de vida, aceitando-se um declínio a uma razão constante, dado por:

$$
1-\mathrm{D}=\left(\mathbf{R}_{\mathfrak{f}} / \mathbf{R}_{\mathbf{i}}\right)^{1 / t}
$$

onde 1-D é a razão de declínio; $R_{f}$ é a proporção de reatores ao fim do período considerado e $R_{\mathbf{i}}$ a proporção no seu ínício; $t$ o tempo em anos decorridos entre o início e o fim.

Como dado inicial foi utilizado o valor $31 \%$, estimado na curva normal reduzida a partir dos resultados de Reis ${ }^{16}$, onde $\bar{X}=8,34$ $\mathrm{mm}$ e $\mathrm{S}=3,38 \mathrm{~mm}$, sabendo-se que os diâmetros das reações ao teste tuberculínico $\mathrm{em}$ vacinados são normalmente distribuídos ${ }^{7}$. O dado final foi obtido pela diferença entre a percentagem total de reatores fortes em escolares com $7 \vdash 8$ anos de idade vacinados no primeiro ano de vida (7\%) e a prevalência de reatores fortes devidos apenas à infecção tuberculosa (4\%) segundo Arantes e col. ${ }^{3,4}$. A razão de declínio assim estimada $(0,7163223)$ foi aplicada inicialmente ao valor $31 \%$ e sucessivamente, até alcançar o valor de $3 \%$ no oitavo ano de vida.

Cálculo da evoluçāo da prevalência de infecção tuberculosa

A evolução da prevalência de infecção tuberculosa do nascimento até o oitavo ano de 
vida foi estimada pela mesma expressão, a partir das proporções de não-infectados, aos 8 anos $(96 \%)$ e ao nascer $(100 \%)$ onde a razão de declínio de não-infecção foi igual a 0,9949102; a prevalência de infectados em cada idade foi obtida subtraindo-se de $100 \%$ os não-infectados. Essa prevalência refere-se ao total a + c na Tabela $2 \times 2$. Sabendo-se que os resultados do teste padronizado em infectados também são normalmente distribuídos ${ }^{8}$, a partir dos dados de Ruffino e col. ${ }^{18}(\bar{X}=16,50$ $\mathrm{mm}$ e $\mathrm{S}=2,82 \mathrm{~mm})$ e de Arantes e col. ${ }^{4}(\bar{X}=$ $17,00 \mathrm{~mm}$ e $S=4,67 \mathrm{~mm}$ ), estimou-se que a sensibilidade do teste anda por volta de $95 \%$. Este percentual aplicado sobre o total a $+\mathrm{c}$ permitiu preencher a casela " $\mathrm{a}$ ".

Cálculo do valor preditivo em relação à infecção

O valor preditivo do teste padronizado em relação à infecção tuberculosa, em crianças vacinadas no primeiro ano de vida, para cada idade desde o primeiro até o oitavo ano, foi entāo calculado para 10.000 vacinados (" $n$ " na Tabela), preenchendo-se as demais caselas e totais por diferenças como se poderá ver no exemplo apresentado na Tabela 3 .

\section{Resultados e Discussão}

Na Tabela 1 é apresentada e evolução da proporção dos reatores fortes do primeiro ao oitavo ano de vida; na Tabela 2 consta a evolução da prevalência estimada da infecção tuberculosa, desde o nascimento até a idade de 9 anos, exclusive. Na Tabela 3 é mostrado o cálculo do valor preditivo positivo do teste tu-

Tabela 1. Evolução da \% de reatores $10 e+\mathrm{mm}$, do final do primeiro ano até a idade de 8 anos, em es. colares vacinados no primeiro trimestre de vida. Razảo de declínio: $1 \cdot d=(3 / 31)^{1 / 7}=0,7163223$.

\begin{tabular}{cc}
\hline $\begin{array}{c}\text { Idade } \\
\text { (anos) }\end{array}$ & $\begin{array}{c}\text { Reaçōes } 10 \text { e }+\mathrm{mm} \\
\text { devidas ao BCG* } \\
(\%)\end{array}$ \\
\hline $0 \vdash 1$ & 31 \\
$1 \vdash 2$ & 22 \\
$2 \vdash 3$ & 16 \\
$3 \vdash 4$ & 11 \\
$4 \vdash 5$ & 8 \\
$5 \vdash 6$ & 6 \\
$6 \vdash 7$ & 4 \\
$7 \vdash 8$ & 3 \\
\hline
\end{tabular}

* Falsos-positivos (FP) para a infecção tuberculosa
Tabela 2 . Evoluçāo da prevalência da infecçāo tuberculosa do nascimento até a idade de 8 anos. Razão de declínio de NĀO infeç̧ão: $1 \cdot d=(96 / 100)^{1 / 8}=$ 0,9949102 .

\begin{tabular}{ccc}
\hline $\begin{array}{c}\text { Idade } \\
\text { (anos) }\end{array}$ & $\begin{array}{c}\text { Não-Infectados } \\
(\%)\end{array}$ & $\begin{array}{c}\text { Infectados } \\
(\%)\end{array}$ \\
\hline $0 \vdash 1$ & 99,49 & 0,51 \\
$1 \vdash 2$ & 98,98 & 1,02 \\
$2 \vdash 3$ & 98,48 & 1,52 \\
$3 \vdash 4$ & 97,98 & 2,02 \\
$4 \vdash 5$ & 97,48 & 2,52 \\
$5 \vdash 6$ & 96,98 & 3,02 \\
$6 \vdash 7$ & 96,49 & 3,51 \\
$7 \vdash 8$ & 96,00 & 4,00 \\
\hline
\end{tabular}

Tabela 3 . Valor preditivo positivo $(\mathrm{VP}+)$ do teste pa. dronizado $(T)$ para a infeção tuberculosa (I) em vacinados no primeiro ano de vida, no ponto médio da faixa etária $01-5$ anos.

\begin{tabular}{|c|c|c|c|}
\hline \multirow{2}{*}{$\begin{array}{c}\text { Teste } \\
(10 e+m m)\end{array}$} & \multicolumn{2}{|c|}{ Infectados } & \multirow[t]{2}{*}{ Total } \\
\hline & Sim & Não & \\
\hline Sim & 144 & 1.600 & 1.744 \\
\hline Não & 8 & 8.248 & 8.256 \\
\hline Total & 152 & 9.848 & 10.000 \\
\hline
\end{tabular}

berculínico para infecção natural, no ponto médio da faixa etária $0 \vdash 5$ anos. $\mathrm{Na}$ Tabela 4 pode ser vista a evolução do valor preditivo positivo em crianças vacinadas no primeiro ano de vida, desse até o oitavo ano.

A avaliação dos atributos (índices estatísticos de desempenho) do teste tuberculínico padronizado em relação à doença tuberculose, cujos critérios de diagnóstico são bem definidos, não apresenta maiores dificuldades. Estudados em 30.885 adultos atendidos em um Centro de Saúde de Ribeirão Preto, SP, a sensibilidade e a especificidade revelaram-se da

Tabela 4. Evolução do VP+ do teste tuberculínico (para a infecçāo tuberculosa), do primeiro ao oitavo ano, em vacinados no primeiro trimestre de vida.

\begin{tabular}{cccc}
\hline $\begin{array}{c}\text { Idade } \\
\text { (anos) }\end{array}$ & $\begin{array}{c}\text { Prevalência } \\
\text { de infecção } \\
\text { tuberculosa } \\
(\%)\end{array}$ & $\begin{array}{c}\text { Prevalência de } \\
\text { reaçōes fortes } \\
\text { devidas ao BCG }\end{array}$ & VP+ \\
\hline $0 \vdash 1$ & 0,51 & $(\%)$ & $(\%)$ \\
$1 \vdash 2$ & 1,02 & 31 & 1,52 \\
$2 \vdash 3$ & 1,52 & 22 & 4,22 \\
$3 \vdash 4$ & 2,02 & 16 & 8,26 \\
$4 \vdash 5$ & 2,52 & 11 & 14,86 \\
$5 \vdash 6$ & 3,02 & 8 & 23,00 \\
$6 \vdash 7$ & 3,51 & 6 & 32,36 \\
$7 \vdash 8$ & 4,00 & 4 & 45,43 \\
\hline
\end{tabular}


ordem de 95,5 e $65,3 \%$ respectivamente, e o valor preditivo do teste positivo foi de $0,42 \%$ com uma prevalência de casos bacilíferos de $0,18 \%^{2}$.

A avaliação direta desses atributos em relação à tuberculose infecção é bem mais difícil: seria necessário contar com métodos diagnósticos mais confiáveis no sentido de discriminar nitidamente infectados e não-infectados, apesar da interferência das reações cruzadas. Razão pela qual se optou por estimativas indiretas, provavelmente aquém da confiabilidade desejável.

A diferença na definição de $t=7$ na Tabela 1 contra $t=8$ na Tabela 2 se deve ao seguinte: no caso da alergia induzida pela vacina, foi observado que em vacinados no período neonatal, seja com dose plena ou com $1 / 2$ dose, não ocorreu declínio até o fím do primeiro ano de vida ${ }^{9}$ e por isso o número de anos decorridos foi a diferença entre 8 e $1=$ 7. Já no caso da evolução da não-infecção (e do seu respectivo complemento que é a infecção) a contagem se inicia no momento zero da vida e por isso $8-0=8$.

A análise da evolução do VP + para a infecção tuberculosa revela que o mesmo é mais baixo no grupo de maior risco ( $1-5$ anos) e que somente no último ano do período esse valor se torna maior do que $50 \%$, ou seja, que a probalidade de uma reação $\geq$ a $10 \mathrm{~mm}$ ser devida ao BK e não ao BCG passa a ser predominante.

$\mathrm{Na}$ faixa etária mais sujeita ao risco de adoecimento primário, especialmente os menores de 2 anos, o VP+ para a infecção por BK é insignificante, mesmo com a elevação do ponto de corte, por exemplo, para $15 \mathrm{~mm}$ (dados não apresentados nos resultados). Deve-se ressaltar que a elevação do ponto de corte, embora aumente a especificidade do teste, acompanha-se de uma correspondente diminuição da sensibilidade,deixando de fora crianças que também seriam passíveis de alguma atenção especial que pudesse diminuirIhes o risco de adoecimento primário.

Entre os comunicantes de casos pulmonares, positivos ao exame baciloscópico do escarro e tossidores potentes, é de se esperar que a grande maioria dos menores de 5 anos esteja infectada por ocasião do diagnóstico, dada a conhecida demora entre o início dos sintomas respiratórios e o início da quimioterapia específica, em nosso meio. A respeito desse tipo de criança, vacinada, comunicante de fonte bacilífera, embora não se tenha de- monstrado que esteja mais sujeita ao risco de adoecimento primário do que as vacinadas não-comunicantes, convêm lembrar que entre os vacinados o risco de complicações extrapulmonares é bem menor ${ }^{5,6,13,22,23}$. Nesse sentido, é preciso salientar que eficácia protetora e efetividade vacinal não significam proporção de protegidos, mas "redução da incidência anual da doença no grupo vacinado em comparação com o grupo não vacinado" ${ }^{\prime 9,20}$.

Considerando-se que o risco daquelas complicaçōes está relacionado com a presença de alterações radiológicas, principalmente parenquimetosas ${ }^{15}$, a radiografia do tórax é o exame complementar mais importante em crianças de baixa idade comunicantes de fontes bacilíferas. Sendo normal, nada a prescrever pois ainda não se demonstrou que a quimioprofilaxia em vacinados traga alguma vantagem adicional. Sendo compatível com lesão tuberculosa primária, a indicação adequada, mesmo na ausência de manifestaçōes clínicas é a quimioterapia.

Isto posto, é possivel concluir que, em áreas com elevada cobertura BCG e baixa prevalência de tuberculose-infecção, o valor preditivo positivo do teste tuberculínico nos primeiros anos de vida é muito baixo, com o que "nos vacinados a prova tuberculínica perde o seu significado".

ARANTES, G.R. [The predictive value of the standard tuberculin test in BCG-vaccinated children]. Rev. Saúde publ., S. Paulo, 26: 264-8, 1992. The applicability of tuberculin test in children under five years of age, BCG-vaccinated during their first year of life, is a controversial matter. With a view to clarifying the subject the predictive positive value of the test in a region of high BCG coverage and low prevalence of tuberculous infection was analysed. From the proportion of strong reactors among infants and school-age children, vaccinated and not unvaccinated, the declining rate of BCG induced allergy and the increment rate of naturally acquired tuberculin sensitivity between the first and the seventh years of life were calculated. Those calculations allowed for the estimation of the respective values for the intermediate ages. The numbers of false positives to be expected were calculated by difference. Knowing the sensibility and the especificity (1 - FP) of the test, the BCG coverage and the prevalence of infection, the predictive values for tuberculos infection were: $1.52 \%, 4.22 \%, 8.26 \%, 14.86 \%$ and $23.00 \%$, respectively from the first to the fifth years of life. Under these conditions, the 
probability of a strong reaction being provoked by $B C G$ is very high, decreasing the applicability of the test both in clinics and epidemiology.

Keywords: Tuberculin test, standards. Predictive value of tests. BCG-vaccine. Tuberculosis in childhood, epidemiology.

\section{Referências Bibliográficas}

1. AMERICAN ACADEMY OF PEDIATRICS. Report of the committee on infectious disesases. $16^{\text {th }} \mathrm{ed}$. Evanston, Ill., 1970.

2. ARANTES, G.R. Planejamento anti-tuberculose: valor preditivo do teste tuberculínico negativo; influência da triagem tuberculinica no valor preditivo da abreugrafia. São Paulo, 1974. [Tese de Doutoramento - Faculdade de Saúde Pública da USP].

3. ARANTES, G.R et al. Influência do BCG aplicado no primeiro ano de vida, no perfil tuberculínico à idade escolar. Medicina (Ribeirão Preto), 23: 232-41, 1990.

4. ARANTES, G.R et al. Estimativa da prevalência da infecção tuberculosa em escolares vacinados com BCG, por meio do método de Bhattacharya. Rev.Saúde públ., S. Paulo, 25: 112-20, 1991.

5. CALVETE, C. et al. Evaluación del efecto protector de la vacinacion con BCG. Bol. Ofic. sanit. panamer., 100: 300-8, 1986.

6. DAM, H.G. ten \& HITZE, K.L. Does BCG vaccination protect the newbom and young infants? Bull. wld Hith Org., 58: 37-41, 1980.

7. EDWARDS, L.B. et al. BCG vaccination. Geneva, World Health Organization, 1953. (WHO Monograph Series, 12).

8. EDWARDS, P.Q. \& EDWARDS, L.B. Story of the tuberculin test from an epidemiologic view-point. Amer. Rev. resp. Dis., 81 (1 pt. 2): 1-47, 1960.

9. GULD, J. Suppurative lymphademitis following intradermical BCG vaccination of the newbom. Brit. med. J., 2: 1048-54, 1955.

10. GULD, J. et al. The duration of BCG induced tuberculin sensitivity in children, and its irrelevance for revaccination: results of two 5 year prospective studies. Bull. Wh Hlth Org., 39: 829-36, 1968.

11. JONCAS, J.H. et al. Interpretation of the PPD skin test in BCG vaccinated children. Can med. Ass.
J., 113: 127-8, 1975.

12. LIFSCHITZ, M. The value of the tuberculin skin test as a screening test for tuberculosis among BCG: vaccinated children. Pediatrics, 36: 624-7, 1965.

13. LUGOSI, L. Trend of children tuberculosis in Hungary 1953 - 1983: quantitative methods for evaluation of BCG policy. Int. J. Epidem., 14: 304-12, 1985.

14. MINISTÉRIO DA SAÚDE. Divisão Nacional de Pneumologia Sanitánia. Manual de normas para o controle da tuberculose. 2 ed. Brasília, 1984. (Série A: Normas e Manuais Técnicos, $n^{2} 13$ ).

15. MOUNT, F.W. \& FEREBEE, S.H. Preventive effects of isoniazid in the treatment of primary tuberculosis in children. New Engl. J. Med, 265: 713-21, 1961.

16. REIS, F.J.C. Alergia tuberculínica e cicatriz vacinal em lactentes que tomaram a vacina BCG injetável quando recém-nascidos. J. Pediat., 52: 23-8, 1982.

17. RIMM, A.A. et al. Basic biostatistics in medicine and epidemiology. New York, Appleton Century Crofts, 1980.

18. RUFFINO-NETTO, A. et al. Aplicação do método de Bhattacharya na análise de resultados do teste tuberculínico. Rev. Saúde públ., S.Paulo, 11: 322-9 . 1977.

19. SMITH, P.G. Retrospective assesment of the effectiveness of BCG vaccination against tuberculosis using the case-control method. Tubercle 62: 23-35, 1982.

20. TOMAN, K. Immunization against tuberculosis: the current state of technical knowledge. In: Regional Seminar on Tuberculosis, $2^{\text {nd }}$, Bogotá, 1972. Washington, D.C., Pan American Health Organization, 1973. p. 28-44 (PAHO - Scientific Publication $\mathrm{n}^{2}$ 265).

21. VECCHIO, T.J. Predictive value of a single diagnostic test in unselected populations. New Engl. J. Med., 274: 1171 - 3, 1966.

22. VOJTEK, $V$. The incidence of tuberculosis meningitis as an indicator of efficacy of BCG vaccination. Tubercle, 41: 272-5, 1960.

23. WUNSCH FILHO, V. Os estudos caso-controle na avaliação da eficácia de vacinas: a eficácia da vacina BCG. São Paulo, 1985. [Dissertação de Mestrado Departamento de Medicina Preventiva da Faculdade de Medicina da USP].

Recebido para publicação em 31/1/1992 Aprovado para publicação em 22/5/1992 